\title{
Low Complexity Video Coding on Block based Singular Value Decomposition (SVD) Algorithm
}

\author{
M.Anto Bennet \\ Associate Professor in \\ Department of ECE, \\ Nandha Engineering \\ College \\ Erode (TN), India.
}

\author{
I. Jacob Reglend \\ Deputy Director - \\ Research,Noorul Islam \\ University, \\ Nagercoil(TN), India.
}

\author{
C.Nagarajan, $\mathrm{PhD}$ \\ Professor in Department \\ of EEE, Muthayammal \\ Engineering College \\ Erode(TN),India.
}

\author{
P.Prakash \\ Department of ECE, \\ Vel Tech Engineering \\ College, Chennai (TN), \\ India.
}

\begin{abstract}
This paper presents analysis of video compression based on block SVD Algorithm. Compression is done to reduce the volume of data to be transmitted, to reduce the bandwidth required for transmission and to reduce the storage requirements. Video is a sequence of still images representing scenes in motion. Current video compression standards like MPEG, H.26x series are highly computationally expensive and hence they are not suitable for real time applications. Current applications like video calling, video conferencing require low complexity video compression algorithms. In addition, the paper investigates the effect of rank in block SVD decomposition to measure the quality in terms of compression ratio and PSNR and also reduce the complexity. The advantage of using the block SVD is the property of energy compaction and its ability to adapt to the local statistical variations of an image.
\end{abstract}

\section{Index Terms}

Block SVD, Low-Complexity video Compression.

\section{INTRODUCTION}

The main objective is to find the video image compression for digital image processing by using the following process

- To improve the efficiency.

- To improve the performance.

- $\quad$ Reduce the cost and time consumption.

- Low Complexity.

\subsection{Video Compression}

Video takes up a lot of space. Uncompressed footage from a camcorder takes up about $17 \mathrm{MB}$ per second of video. Because it takes up so much space, video must be compressed before it is put on the web. "Compressed" just means that the information is packed into a smaller space. An overview of video compression formats is described below.

\subsubsection{Jpeg}

For single-frame image compression, the industry standard with the greatest acceptance is JPEG (Joint Photographic Experts Group). JPEG consists of a minimum implementation (called a baseline system) which all implementations are required to support, and various extensions for specific applications. JPEG has received wide acceptance, largely driven by the proliferation of image manipulation software which often includes the JPEG compression algorithm in software form as part of a graphics illustration or video editing package. JPEG compressor chips and PC boards are also available to greatly speed up the compression/decompression operation. JPEG compressor chips and PC boards are also available to greatly speed up the compression/decompression operation.

The main disadvantage of JPEG algorithm the balkiness appears when compression ratio is pushed too high.

\subsubsection{Motion JPEG}

MPEG is the "Moving Picture Experts Group", working under the joint direction of the International Standards Organization (ISO) and the International Electro-Technical Commission (IEC). This group works on standards for the coding of moving pictures and associated audio. MPEG involves fully encoding only key frames through the JPEG algorithm (described above) and estimating the motion changes between these key frames. Since minimal information is sent between every four or five frames, a significant reduction in bits required to describe the image results. Consequently, compression ratios above 100:1 are common. The decoder puts the images back into display sequence. It takes on the order of 1.1-1.5 billion operations per second for real-time MPEG encoding. Now newer silicon is enabling some forms of MPEG compression on the desktop in real time.

A digital video sequence can be represented as a series of JPEG pictures. The advantages are the same as with single still JPEG pictures - flexibility both in terms of quality and compression ratio.

The main disadvantage of motion JPEG is that since its uses only a series os still pictures it makes no uses of video compression techniques. The result is a lower compression ratio for video sequences compared to "real" video compression technique lines MPEG.The benefits is its robustness with no dependency between the frames, which means that, for example, even if one frame is dropped during transfer, the rest of the video will be un-effected.

\subsubsection{Motion JPEG 2000}

As with JPEG and Motion JPEG, JPEG 2000 can also be used to represent a video sequence. The advantages are equal to JPEG 2000 , i.e., a slightly better compression ratio compared to JPEG but at the price of complexity. The disadvantages of motion JPEG 2000 is generally considered not as good as a motion JPEG steam, and motion JPEG 2000 has never been any success as a video compression technique.

\subsubsection{H.261/H.263}

H.261 was developed in 1990 by the international Telecommunication union (ITU) developed the H.261 standard for the data rates that are multiples of 64kbps. H.261 standard uses motion compensated temporal prediction. Its supports two resolution, namely common interface format (CIF) with a frame size of $352 \times 288$ and quarter CIF (QCIF) with a frame size of $172 \times 144$. 
H.263 was developed by the international Telecommunication union (ITU) in 1996.It uses an encoding algorithm called the test model (TMN), which is similar to that used by H.261 but improved performance and error recovery leading to higher efficiency. It's optimized for coding at low bit rates provides the same quality as H.261 but with half the number of bits. H.263 is like H.261, is not suitable for usage in general digital video coding. However, H.261 and 263 are a bit contradictory since they both lack some of the more advanced techniques.

The H.261 and H.263 are not International Standards but only Recommendations of the ITU. They are both based on the same technique as the MPEG standards and can be seen as simplified versions of MPEG video compression. They were originally designed for video-conferencing over telephone lines, i.e. low bandwidth. However, it is a bit contradictory that they lack some of the more advanced MPEG techniques to really provide efficient bandwidth use. The conclusion is therefore that H.261 and H.263 are not suitable for usage in general digital video coding.

\subsubsection{MPEG-1}

MPEG-1 , the Coding of Moving Pictures and Associated Audio for Digital Storage Media at up to about $1.5 \mathrm{Mbps}$, is an International Standard ISO-11172, completed in October, 1992. MPEG-1 is intended primarily for stored interactive video applications (CD-ROM); with MPEG-1, one can store up to 72 minutes of VHS quality ( 640 x $480 \mathrm{~s} 30 \mathrm{fps})$ video and audio on a single CD-ROM disk. MPEG-1 can deliver full-motion colour video at 30 frames per second from CDROM. Because audio is usually associated with full motion video, the MPEG standard also addresses the compression of the audio information at 64, 96, 128, and $192 \mathrm{kbps}$ and identifies the synchronization issues between audio and video by means of time stamps. The first volume application for MPEG-1 decodes chips (from C-Cube Microsystems) was a Karaoke entertainment system by JVC.

MPEG-1 video compression is based upon the same technique that is used in JPEG. In addition to that it also includes techniques for efficient coding of a video sequence. It has no direct provision for interlaced video applications.

\subsection{6. $M P E G-2$}

MPEG-2 is the "Generic Coding of Moving Pictures and Associated Audio." The MPEG-2 standard is targeted at TV transmission and other applications capable of 4 Mbps and higher data rates. MPEG-2 features very high picture quality. MPEG-2 supports interlaced video formats, increased image quality, and other features aimed at HDTV. MPEG-2 is a compatible extension of MPEG-1, meaning that an MPEG-2 decoder can also decode MPEG-1 streams. MPEG-2 audio will supply up to five full bandwidth channels (left, right, center, and two surround channels), plus an additional lowfrequency enhancement channel, or up to seven commentary channels. The MPEG-2 systems standard specifies how to combine multiple audio, video, and private-data streams into a single multiplexed stream and supports a wide range of broadcast, telecommunications, computing, and storage applications.

The MPEG-2 is suitable for TV broadcast applications and high quality archiving applications. It's however designed for the internet, as it requires too much bandwidth.

\subsection{7. $M P E G-3$}

MPEG-3 was merged into MPEG-2 and no longer exists. The next version of the MPEG standard, MPEG-3 was designed to handle HDTV, however, it was discovered that the MPEG-2 standard could be slightly modified and then achieve the same results as the planned MPEG-3 standard. Consequently, the work on MPEG-3 was discontinued.

The next version of the MPEG standard, MPEG-3 was designed to handle HDTV, however, it was discovered that the MPEG-2 standard could be slightly modified and then achieve the same results as the planned MPEG-3 standard. Consequently, the work on MPEG-3 was discontinued.

\subsubsection{MPEG-4}

MPEG-4 is for very low bit rate coding and is scheduled to be available in draft form in late 1997 or early 1998. MPEG-4 and H.324 are likely to merge. MPEG-4 began life with a mandate by the Moving Picture Experts Group to create audiovisual coding schemes at very low bit rates for wireless telecommunications. The scope of the work has been expanded to target a broader spectrum of applications. MPEG-4 completion is scheduled for 1998. MPEG-4 is an inclusive superset of MPEG-1 and MPEG- 2 and is based on a common communication language to describe tools, algorithms, and profiles necessary for coding of objects, rather than standardizing a coding algorithm. MPEG-4 specs will include methods for combining synthetic scenes or objects with natural scenes, and for coding and manipulating them without first converting the objects into video frames. MPEG4 would also code audio and video objects at their native resolutions, supporting content-based manipulation and bitstream editing without the need for transcending.

Scalability is required for video transmission over heterogeneous networks so that receiver obtain a full resolution display. The MPEG -4 provides a higher coding efficiency for storage and transmission of audio-visual data at very low bit rates. About $5-64 \mathrm{kbps}$ is used for mobile or PSTN video applications and at up to $2 \mathrm{Mbps}$ for TV/film applications.

\subsection{9. $M P E G-7$}

It was approved in July 2001 to standardize a language to specify description schemes. The MPEG -7 is a different kind of standard as it a multimedia content description standard and does not deal actual encoding of moving pictures and audio. MPEG-7 uses XML to store metadata and it can be attached to time code in order to tag particular events in a stream. Although , MPEG-7 is independent of the actual encoding technique of the multimedia, the representation that is in defined with in MPEG -4, i.e., The representation of a audio - visual data in terms of objects, is very well suited to the MPEG-7 standard. MPEG-7 is relevant for video surveillance since it could be used for example to tag the contents and events of video streams for more intelligent processing in video management software or video analytic applications.

MPEG-7 addresses this problem as it caters for both real-time and non-real time applications and enables retrieval of multimedia data files from the Internet. If the available network bandwidth is limited, or if a video is to be recorded at a high frame rate and there are storage space restraints, MPEG may be the preferred option. It provides a relatively high image quality at a lower bit-rate (bandwidth usage). Still, the 
lower bandwidth demands come at the cost of higher complexity in encoding and decoding, which in turn contributes to a higher latency when compared to motion.

\subsubsection{H.264/AVC}

H.264/AVC was approved by ITU-T in March 2003. H.264 is the latest generation for video encoding. This initiative has many goals. It should provide good video quality at substantially lower bit rates than previous standards and with better error robustness - or better video quality at an unchanged but rate. The standard is further designed to give lower latency as well as better quality for higher latency. In addition, all these improvements compared to previous standards were to come without increasing the complexity of design so much that it would be impractical or expensive to build applications and systems.

An additional goal was to provide enough flexibility to allow the standard to be applied to a wide variety of applications: for both low and high bit rates, for low and high resolution video, and with high and low demands on latency. Indeed, a number of applications with different requirements have been identified for H.264:

- Entertainment video including broadcast, satellite, cable, DVD, etc (1-10 Mbps, high latency)

- $\quad$ Telecom services (<1Mbps, low latency)

- Streaming services (low bit-rate, high latency)

The DVD players for high-definition DVD formats such as HD-DVD and Blu-ray support movies encoded with H.264.

The comparison of proposed block based singular value decomposition [SVD] the time complexity is greatly reduced and also accuracy is high when compare with 2D-SVD.The block SVD algorithm which provides higher PSNR at the same bit rate [1]. The high compression ratio so computational complexity also to be increased [2]. A video surveillance compression system the main problem is to increase in computational complexity, high energy consumptions, short batter life [3]. The mobile wireless technology has increased its data capacity, but the unpredictable nature of mobile channels still results in severe fading effects. Here increase in CPU cycles at encoder end, as more number of computations had to be done before generating the final optimized bit stream. There was increase in padding bits in virtually partitioned macro blocks. Current work includes optimizing the bit usage and modifying the motion vector sort algorithm to support random errors [4]. Variable block-size motion estimation (VBSME) has become an important video coding technique, but it increases the difficulty of hardware design[5]. ]. Our ICR system proved to be efficient and reduced the time required for processing process [6]. H.263 provides better PSNR results than MWDR at high bit rate; their coding efficiencies are comparable at higher frame rates. The visual qualities of both codec's arc comparable. As expected, H.264 provides the best quality among all codec's [7]. High bandwidth requirements for transmission and low resilience to errors due to missing frames. The modification provides higher compression, maintains perceptual visual quality and reduces video degradation due to missing frames or missing packets [8]. The proposed algorithm leads to a satisfactory battery life, and that for applications where battery life of months instead of weeks is required, fully software solutions are not adequate, but it is necessary to implement some parts of the signal processing algorithms in low-power (possibly reconfigurable) hardware such as FPGA or ASIC [9].

The facsimile-based video-compression systems are well suited for software-only video applications with stringent latency requirements such as teleconferencing and distance learning. In particular, the direct subtraction system eliminates the need for costly video boards by eschewing motion estimation/compensation, replaces 2-D transform coding with facsimile-based coding, and nonetheless provides a competitively high compression ratio to facilitate transmission [10]. Optimum bit rate pyramid coding method the quality of the reconstructed image is ranged from lossless image compression. It's used for low bit requirement application like visual telephone and telebrowsing only [11].

During the past decades digital techniques have made numerous inroads into different fields, mainly thanks to the large size of the data processing and consumer electronics markets. This was possible thanks to the stand-alone nature of many of these equipment and their rapid obsolesce that forced users to a rapid turnover. Not equally so in the field of telecommunications, here meant to include broadcasting, where replacement of even upgrade of the infrastructure takes understandably a much longer time [12]. The PIT algorithm is encoding this sequence by transmitting from smallest to the largest size .so it's take longer time for the encoding process [13]. The main drawback of JPEG is blackness appears in the images when the compression ratio is pushed too high [14].The design of block codes of given length $n$ to have small error probability is a more difficult and more interesting problem for correlated sources than for a single source [15].

Image compression plays an important role in multimedia application, such as image storage and transmission. The basic goal of image compression is to represent an image with minimum number of bits. When used to convey multimedia transmissions, video streams contain a huge amount of data that requires a large bandwidth and subsequent storage space. As a result of the huge bandwidth and storage requirements, digital video is compressed in order to reduce its storage or transmitting capacity. This technology (video compression) reduces redundancies in spatial and temporal directions. Spatial reduction physically reduces the size of the video data by selectively discarding up to a fourth or more of unneeded parts of the original data in a frame. Temporal reduction, Inter-frame delta compression or motion compression, significantly reduces the amount of data needed to store a video frame by encoding only the pixels that change between consecutive frames in a sequence. Several important standards like Moving Picture Experts Group (MPEG) standard, H.261, 263 and 264 standards are the most commonly used techniques for video compression.

\section{SINGULAR VALUE DECOMPOSITION}

In Linear Algebra, Singular Value decomposition is nothing but factorization of a matrix in the form

$$
\mathrm{A}=\mathrm{U} \Sigma \mathrm{V}
$$

Where $\mathrm{U}$ and $\mathrm{V}$ are orthogonal matrices and $\square$ is a diagonal matrix.

$A=($ Orthogonal) (Diagonal) (Orthogonal) 


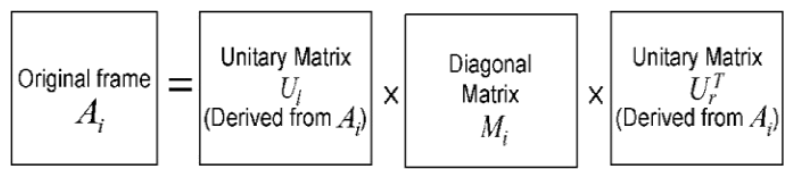

Fig .1.SVD decomposition.

Fig .1.shows the SVD decomposition process. The columns of $\mathrm{U}$ are composed of the eigenvectors of AT, the columns of V are composed of the eigenvectors of ATA. The diagonal values of $\Sigma$ are nothing but the square roots of the non-zero eigenvalues of both AAT and ATA.2D-SVD is an extension of the above mentioned 1DSVD.2D-SVD has been extensively studied for computer vision. The main drawback of 1D-SVD for image compression applications is that, even though it provides the most energy compaction (the coefficients are present only along the diagonal), it requires the transmission of the two eigenvector matrices for each block. This incurs very high overhead thereby reducing the compression efficiency. In 2D-SVD, the eigenvector matrices are extracted from a group of blocks. Therefore, the two matrices have to be transmitted only for a group of blocks. This results in higher compression. The block SVD algorithms are used to achieve higher PSNR at the same bit rate.

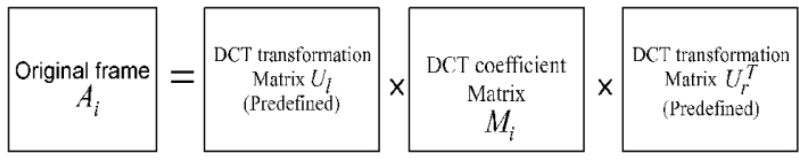

Fig. 2.DCT decomposition.

For the DCT one fixed matrix is used, thus Ul=Ur. The DCT decomposition is shown in Fig.2. Since the transformation matrix is fixed, we can simply represent Ai with Mi.

The main problem for block-based 1-D SVD coding is that, although its coefficient matrix contains the fewest nonzero coefficients compared with other transforms, its and transformation matrices are not fixed and, hence, need to be sent to the decoder for each frame. As a result, the overall coding efficiency for the block-based 1-D SVD coding is not very promising.

\section{2D SVD PROCESS:}

An image or a video frame can be divided into $\mathrm{m} \times \mathrm{m}$ non overlapping blocks. The 2D SVD decomposition is shown in Fig. 3.

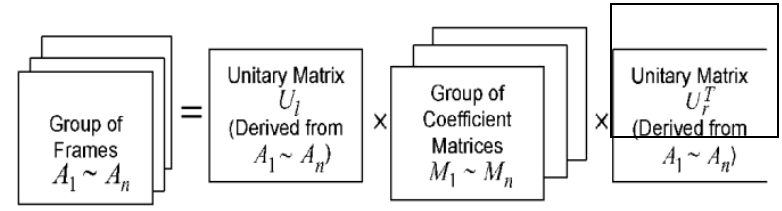

Fig. 3.Two-dimensional SVD decomposition.

The basic algorithm to find the coefficient matrix is given below:

1) Given a group of frames $A_{1} \ldots \ldots A_{n}$ find the mean frame Amean. Obtain the mean subtracted frames i.e. $A_{i}{ }^{1}=A_{i}-A_{\text {mean }}$

2) For any GOB (Group of Blocks), $B$ in the meansubtracted frames, we denote as each block in B as $b_{1} \ldots \ldots b_{n}$. For each GOB, find the row-row and column-column covariance matrices $\mathrm{F}$ and $\mathrm{G}$

$$
\mathrm{F}=\sum_{\mathrm{i}=0}^{\mathrm{n}} \mathrm{bib} \mathrm{T}
$$

$$
\mathrm{G}=\sum_{\mathrm{i}=0}^{\mathrm{n}} \mathrm{bT} \text { bi }
$$

$\mathrm{U}_{1}$ and $\mathrm{Ur}$ are made up of the $\mathrm{k}$ principal eigenvectors of $\mathrm{F}$ and $\mathrm{s}$ principal eigenvectors of respectively. It has been reported in that the lowest mean squared error is obtained when $\mathrm{k}=\mathrm{s}=1$.

3) The coefficient matrix $M$ is obtained using the formula

$$
\mathrm{M}=\mathrm{U}_{1}^{\mathrm{T}} \mathrm{b}_{\mathrm{i}} \mathrm{U}_{\mathrm{r}}
$$

it is to be noted that Miis not a diagonal Matrix. However, most of the non-zero coefficients willbe located close to the principal diagonal.

4) To get back the original frame, the mean subtracted block is first obtained using the Formula

$$
\mathrm{bi}=\mathrm{U}_{\mathrm{l}} \mathrm{MiUr}^{\mathrm{T}}
$$

The near optimal approximation of each block is obtained using

$$
\mathrm{b}_{\mathrm{i}}{ }^{\mathrm{c}}=\mathrm{b}_{\mathrm{i}}{ }+\mathrm{b}_{\text {mean }}
$$

where bmean is the corresponding block in the mean frame.

\section{PROPOSED TECHNIQUE:}

The application of the algorithm proposed in yielded the following observation. The Fig. 4 shows the video compression based on block SVD algorithm. The various steps are involved in the process of Block SVD video compression techniques. The sequence of process is input video sequence, Frames, Encoder, Block SVD, decoder and finally will get the Compressed output video sequence.

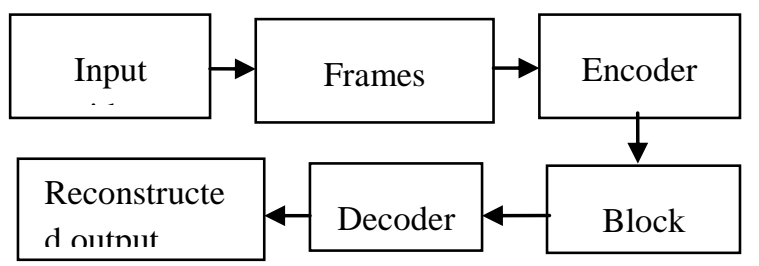

Fig.4. Video compression based on Block SVD method.

In video compression the images are called as the frames. Initially, the input video is divided into group of frames. Here Pre-processing algorithms used improve on the performance of a video compression system. Its increases compression efficiency and attenuates coding artifacts. The size of the video frames is high means it's not display for some applications. So the frames are reconstructed to the standard size. The purpose of an encoder is to reduce the number of bits in the original image. Each frame is divided into several blocks. The block sizes are $\mathrm{m} \times \mathrm{m}$ non-over lapping group of blocks (GOB). Block SVD algorithm used for data reduction technique. The group of co-efficient matrices is optimized for block SVD. According to the experiment minimum mean square error is achieved. The decoder is the reverse steps of encoder process. The group of information is decoded in the function of decoder. Here the group of information size is small, successfully received. The Each frame are decode independently, just like an intra frame video codec. The sampling and quantization techniques are used to remove the error in decoder process. Finally get the quality of 
the compressed reconstructed video. It's the purpose of reducing storage space in the memory.

\subsection{ENCODER}

In block SVD process encoder are used for the purpose of compressed technique. The purpose of an encoder is to reduce the number of bits in the original image. Each frame is divided into several blocks. The block sizes are $\mathrm{m} \mathrm{x} \mathrm{m} \mathrm{non} \mathrm{-}$ over lapping group of blocks (GOB).

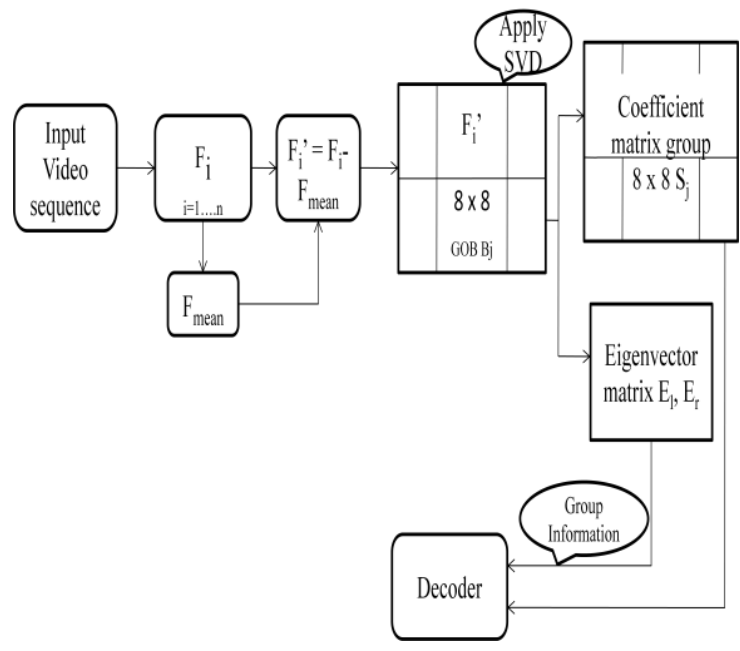

Fig .5. Block SVD Encoder Process

The Fig.5 shows the proposed Block SVD encoder process. Consider the ith frame denoted as Fi. The mean frame Fmeanis found from $\mathrm{n}$ frames andthe mean subtracted frames is obtained, i.e. Fi' $=\mathrm{Fi}$ - Fmean. Then the mean-subtracted group of pictures(GOP) is divided into $8 \times 8$ group of blocks(GOBs) where each block in B is denoted as $\mathrm{B} 1, \ldots ., \mathrm{Bn}$. SVD is applied for the jth GOB to obtain the corresponding eigenvector matrices $\mathrm{El}$ andEr and the group of $8 \times 8$ coefficient matrices S1...Sn. The eigenvector matrix contains eight eigenvectors. The eigenvectors with small subindices are more important since they correspond to the top left coefficients which contain more information. First, we extract the mean frame from the GOP with frames and compress it with JPEG standard with a high quality factor. Then, we divide the mean-subtracted GOP into 8x8 GOBs. The group of co-efficient matrices is optimized for block SVD.Finally co-efficient matrixes are added to form the group of information. According to the experiment minimum mean square error is achieved.1-D SVD video is divided into each blocks and then each block is coded with 1-D SVD. So, the large co-efficient are needed for the process. Here we proposed system $50 \%$ co- efficient is needed only.

\subsection{DECODER}

The decoder is the reverse steps of encoder process. The group of information is decoded in the function of decoder. The main frame and Eigen vector are extracted. We get reconstructed video frame without the mean frame by inverse SVD. Here the group of information size is small, successfully received. The Each frame are decode independently, just like an intra frame video codec.

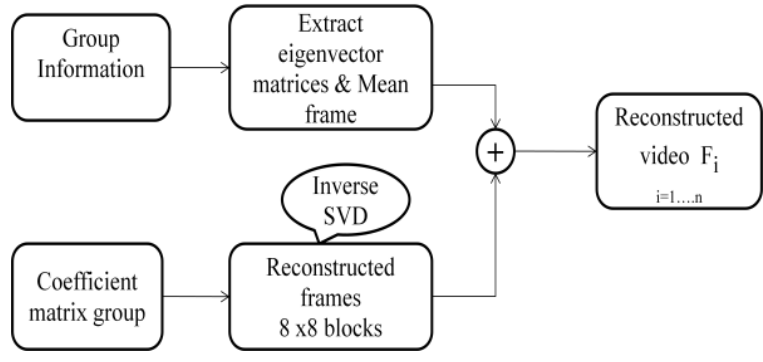

Fig.6. Block SVD Decoder Process

The decoder part, as illustrated in Fig.6, basically carries out the reverse steps of the encoder. First, the GI is decoded; the mean frame and eigenvector matrices are extracted. Then, we get the reconstructed frames without the mean frame by inverse SVD as, where is the number of $8 \times 8$ blocks in each $n$ frame. Finally, by adding the reconstructed mean frame, we obtain the reconstructed frame. In this way, once the GI, whose size is small, is successfully received, we are able to decode each frame independently, just like an intraframe video codec. This interframe independence is a very desirable property since the problems such as error propagation and difficulties in random frame access are all due to the interframe dependence. A lot of work in the literature has been done to deal with these problems by introducing complex codec structure or transmission protocol. Taking inverse Block SVD to the process. Finally we will get the compressed reconstructed video sequence.

\section{Proposed Algorithm}

1) For videos with low motion, increase in GOP increased the compression ratio while maintaining the PSNR almost constant. This is because the percentage of GI to the coefficients is reduced, while maintaining the energy compaction property of SVD.

2) For videos with high motion, increase in GOP decreased the compression ratio and the PSNR also reduced. This is because the energy is distributed over a large number of coefficients thereby reducing the coding efficiency.

From the above observation it is clear that, for videos with low motion, a large GOP can be used and for videos with high motion a very low value of GOP must be used to maintain the compression ratio and the PSNR. The proposed algorithm is based on this inference:

1) First, the incoming images are divided into $8 \times 8$ non-overlapping blocks, $\mathrm{Bj}$ where $\mathrm{j}=0 .$. heightxwidth

2) For each block $\mathrm{Bj}$, the difference between the current block and the corresponding block in the 10th next frame is computed. The sum of these differences is computed.

3) If this sum is less than the threshold th, then the GOP value is set to GOPhigh. If it is greater than the threshold, then it is set to GOPlow. For each block, a group related information is first sent to the decoder. In this GI, the first bit set to 1 if GOP $=$ GOPhigh and it is set to 0 if GOP = GOPlow. 
4) The mean block b mean, is calculated using the formula

$$
\mathrm{b}_{\text {mean }}=\frac{1}{G O P} \sum_{i=1}^{G O P} b i
$$

The mean block is then encoded using the JPEG algorithm. It is to be noted that, GOPhigh is chosen to be an integral multiple of GOPlow. If the GOP is GOPlow then GOPhigh GOPlow number of mean blocks are sent.

5) Block SVD mentioned above is used to obtain the corresponding eigenvector matrices $\mathrm{U}_{\mathrm{j}}^{\mathrm{j}}$ and $\mathrm{U}_{\mathrm{r}}^{\mathrm{j}}$ the group of $8 \times 8$ coefficient matrices $M_{1}{ }^{j} \ldots \ldots M_{n}{ }^{j}$

6) The eigenvectors $U_{1}{ }^{j}=\left(U_{1}{ }^{j} \ldots . . U_{8}{ }^{j}\right)$ and

$U_{r}^{j}=\left(U_{r}^{j} \ldots . . U_{r}^{j}\right)$ are encoded using the Vector Quantization strategy. Code books of length 256, $256,128,128,128,64,64$, and 32 are used to quantize the eigenvectors respectively. Eigenvectors derived by applying the Block SVD algorithm to some standard sequences are used to learn the codebooks. The LBG algorithm is used to learn these codebooks. These codebooks are stored in both encoder and decoder, therefore the coding of the eigenvectors is achieved using the least amount of bits possible.

7) There is no need to transmit all the obtained eigenvectors. Let $=\mathrm{M}_{\mathrm{i}}^{\mathrm{j}}(\mathrm{x}, \mathrm{y})$ denote the coefficient value at frame $\mathrm{i}$ of block $\mathrm{j}$ at position $(\mathrm{x}, \mathrm{y})$. If Xmax denotes the maximum $x$ position of the nonzero coefficients for block $\mathrm{j}$ and frames $\mathrm{i}$... $\mathrm{N}$ and Ymax denotes the maximum y position of the nonzero coefficients, then we need to transmit only the eigenvectors $\left(\mathrm{U}_{1}{ }^{\mathrm{j}} \ldots . . \mathrm{U}_{\mathrm{X} \text { max }}{ }^{\mathrm{j}}\right)$ and $\left(\mathrm{V}_{1}^{\mathrm{j}} \ldots . . \mathrm{U}_{\mathrm{Ymax}}{ }^{\mathrm{j}}\right)$. Six bits are included in GI, to denote the number of eigenvectors sent per GOB.

8) The coefficient matrices $\mathrm{M}_{1}{ }^{\mathrm{j}} \ldots . \mathrm{M}_{\mathrm{n}}{ }^{\mathrm{j}}$ are then quantized, zigzag read and entropy encoded using the JPEG algorithm.

\section{EXPERIMENTAL RESULTS}

In our proposed system we use the Block SVD algorithm, better computational complexity and temporal redundancy reduction. For 1-D SVD more co-efficient are required for the process, 2-D SVD needs only about 50\% of co-efficient compared with 1-D SVD. The energy compaction property of Block SVD coefficient matrices, high coding efficiency is achieved. The comparison of the proposed Block SVD coding scheme with the existing relevant non-ME-based lowcomplexity code shows its advantages and potential in applications. By using the block singular value decomposition the time complexity is greatly reduced when compare with 2D-SVD.The block SVD algorithm, which provides higher PSNR are present at the same bit rate.

\subsection{Input Video Frames}

Fig 7 shows the input video frames. In a video, the images called as frames are streamed at the rate of 25-30 frames per second (fps). Video is characterized by huge amount of data. The main drawback of uncompressed video frames required storing more memory and also transmission of raw video requires huge bandwidth. . To reduce the transmission bandwidth and the storage requirements, video compression is done.

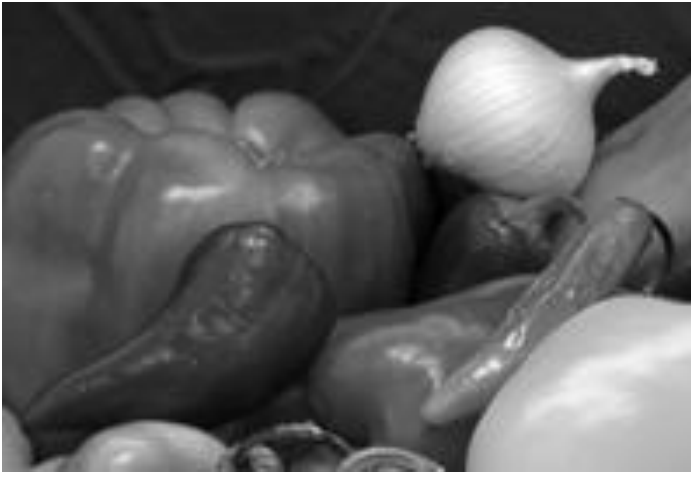

Fig.7. Input video frames

\subsection{DCT Decomposition}

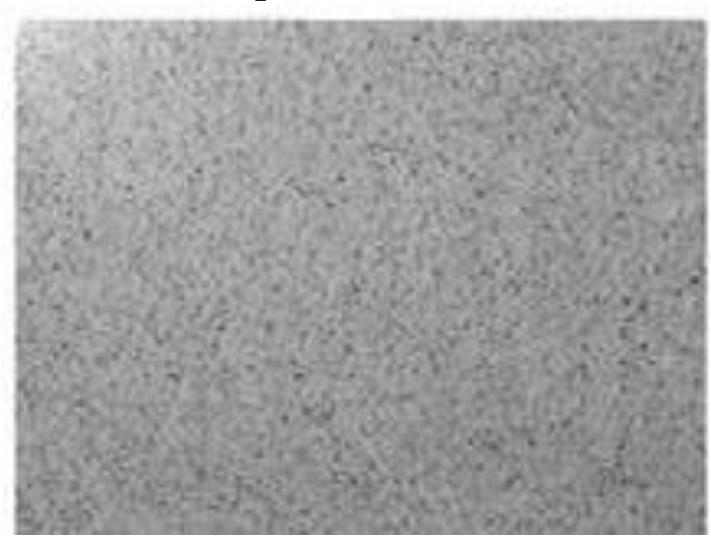

Fig. 8 . DCT decomposition

The fig 8 shows the DCT decomposition for video compression based on Block SVD algorithm. The DCTbased encoder worked by segmentation the image into $8 * 8$ blocks. Each block makes its way through each processing step, and yields output in compressed form into the data stream. As image pixels are highly correlated, the DCT achieves data compression by concentrating most of the signal in the lower spatial frequencies. For a typical $8 * 8$ sample block from a typical source image, most of the spatial frequencies have zero or near-zero amplitude and need not be encoded. In principle, the DCT introduces no loss to the source image samples; it transforms them to a domain in which they can be more efficiently encoded. Then for $\mathrm{M}^{*} \mathrm{~N}$ image a single column matrix is generated and their square is calculated and store in reconstructed matrix.

\subsection{Image Compression With Singular Value Decomposition}

The SVD matrix decomposition is extensively used in Mathematics. It appears in fields related directly with algebra, such as least squares problems or the calculus of the matrix rank. Its usefulness in applications concerning image processing has also been evaluated. Among these applications we can mention patron recognition, secret communication of digital images, movement estimation, classification and quantization. The interest of this transformation comes from the fact that by using it we obtain easily the best approximation of a given rank in terms of the 2-norm. Singular Value Decomposition of a matrix is Calculated By:

$[\mathrm{u}, \mathrm{s}, \mathrm{v}]=\operatorname{svds}(\mathrm{red}$, singvals $)$; 
Imred $=$ uint $8(\mathrm{u} * \mathrm{~s} *$ transpose $(\mathrm{v}))$;

For $\mathrm{M} * \mathrm{~N} * 3$ Image

Where $\mathrm{u}=\mathrm{N} *$ singvals, $\mathrm{s}=$ singvals*singvals, $\mathrm{v}=\mathrm{M} *$ singvals

singvals is the number of largest singular values (positive integer) This equation reconstruct matrix for red plane. Similarly green plane and blue plane reconstructed and compressed image reconstructed by combining these three planes R, G, B. We can see changes in images by changing the singvals values, size increase with increase in signvals value and quality also improve.

\subsection{Inverse Svd Applied Image}

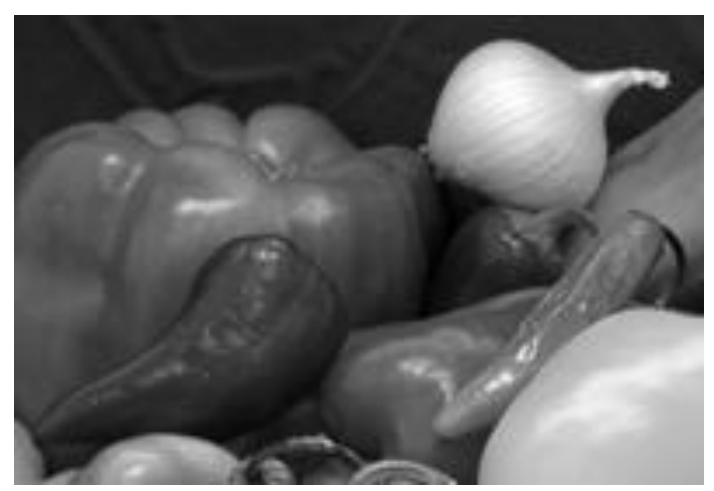

Fig .9 . Inverse svd appiled input video frames.

The fig 9 shows the inverse SVD applied input video frames for video compression methods.Inverse SVD are used to get the images from input SVD algorithm.

\subsection{Performance Analysis To Various Video Coding}

The main aim of this paper is to improve the PSNR rate and reduce the complexity .Here compare the different video codec's based on peak-signal-to-noise ratio graph shown below.

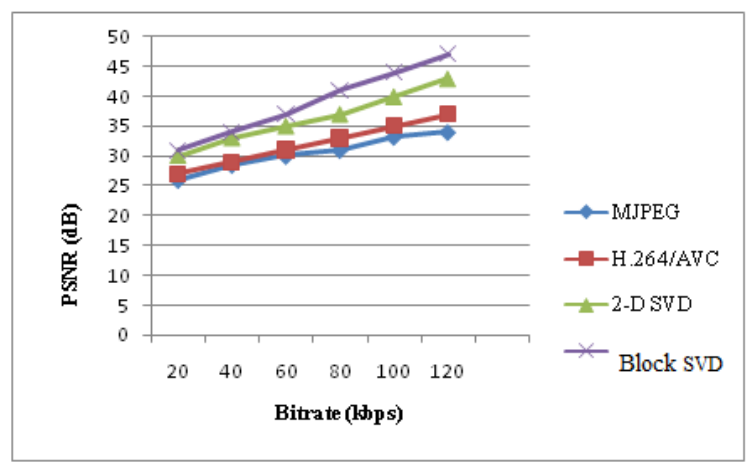

Fig.10. Analysis the various video coding performances

Fig.10. shows the comparison of existing and proposed methods of different video codec's based on their performance in terms of peak-signal-to-noise ratio where the proposed Block SVD video coding technique provides high PSNR value.

\section{CONCLUSION}

The video compression based on block SVD algorithm was implemented in MATLAB. The proposed block SVD video coding scheme are used to achieve higher coding efficiency in terms of peak signal-to-noise ratio(PSNR) by 3-7 dB for the given video sequence compared with existing hybrid video codec's .Video compression is gaining popularity since storage and network bandwidth requirements are able to be reduced with compression. Many algorithms for video compression which are designed with a different target in mind have been proposed. Video compression such as H.261, 263 and 263+, MPEG-1, 2, 4, 7 and H.264. Most recent efforts on video compression for video have focused on scalable video coding. The primary objectives of on-going research on scalable video coding are to achieve high compression efficiency high flexibility (bandwidth scalability) and/or low complexity. Due to the conflicting nature of efficiency, flexibility and complexity, each scalable video coding scheme seeks tradeoffs on the three factors. Designers of video services need to choose an appropriate scalable video coding scheme, which meets the target efficiency and flexibility at an affordable cost and complexity. The biggest advantage of hybrid video coding technique is that it is designed for real time transmission. Such as better visual quality and this video coding method is suitable for applications such as mobile video phone, video surveillance systems, wireless video cameras, and disposable video cameras etc. The low complexity video coding based on Block SVD algorithm used to get reconstructed video. The higher PSNR are present at the same bit rate Further we can analysis to reduce the time complexity reduction will be a future challenge of the video compression process based on block SVD Algorithm.

\section{REFERENCES}

[1] ZhouyeGu, WeisiLin,Bu-sung Lee, ChiewTong Lau( 2012), " Low - Complexity Video Coding based On Two-Dimensional Singular Value Decomposition", IEEE Transactions on Image Processing, vol. 21, no. 2, pp 674-687.

[2] Abomahara . M , Khalifa .O .O , Zaidan .A .A (2010) , “ Video Compression Techiques an Overview “, Journal of applied sciences 10 (16) - pp. $1834-1840$ ISSN $1812-5654$ at asian network for scientific information.

[3] Liu .L. -M, Li .Z and Delp .E.J (2009) , “ Efficient and Low - Complexity Surveillance Video compression using backward - channel aware Wyner Ziv Video Coding," IEEE Trans. Circuits Syst. Video Technol., vol. 19, no. 4, pp. 453-465.

[4] Premkumar Elangova , Gaoyong Leo , Geoff Lawday ( 2007), "Structurally Efficient Video Codec for Wireless Mobile Appilication ," ITNG '07 International Conference on Information Technology.

[5] Ching yeh chen, Shao yi chien, yi - wen huang , Tung Chien Chen (2006), "Analysis and Architure Design of Variable Block Size Motion Estimation For H.264/AVC ," IEEE Trans.Circuits System., Vol.53, no.2.

[6] Dipiti Deodhare, NNR Ranga suri , Amit . R (2005),“ Preprocessing and Image Enhancement Algorithms for a Form- based Intelligent Character Recognition 
System," International Journal of Computer Science and Applications, Vol .2, No.2. pp.131 -144.

[7] Yee L. Law and Truong Q. Nguyen ( 2004 ) ," Motion Wavelet Difference Reduction (MWDR) Video Codec ,'International Conference on Image Processing, pp.2303-2306.

[8] Sanmati Kamath \& joel R. Jackson ( 2004 ), “ Low Bit Rate Motion JPEG Using Differential Encoding," IEEE, pp . $1723-1726$.

[9] Enrico Magli , Massimo Mancin , Luca Merello (2003), " Low Complexity Compression for Wireless Sensor Network," IEEE Trans. Centre for Multimedia Radio Communications., pp.585 - 588 .

[10] Yi - Jen Chiu Toby Berger (1999), " A Software Only Video codec Using Pixelwise Conditional Differential Replenishment And Perceptual Enhancements ,"IEEE. Trans. Circuits And Systems For Video Technology, Vol .9, no.3, pp. $438-450$.
[11] Al - Asmari . Kh (1995) ," Optimum bit rate pyramid coding with low Computational and memory requirements," IEEE Trans. Circuit and Syst . For video Tech., Vol.5, No.3.

[12] Leonardo Chiariglione (1995 ), " The Development of an Integrated Audiovisual MPEG," IEEE Trans . Vol .83, No .2.

[13] Hwang .W and Derin . H (1995), "Multi resolution multiresource progressive image transmission ," IEEE Trans.Image Processing, Vol.4, No.3.pp .1128-1140

[14] Pennebaker .W . B and Mitchel .J .L (1993)," JPEG still image data compression Nostrand Reinhold, New York. standard," Van

[15] Slepian . D and Wolf . J. K. (1973) ," Noiseless coding of correlated information Sources ," IEEE Transcation on Information Theory, Vol . 19, pp .471 490. 